Isto é, não é caso de responsabilidade jurídica, pela qual o juiz que pratica certa conduta sofre sanção prevista em lei. Trata-se aqui de uma atuação no âmbito estrito do exercício da jurisdição, e nosso sistema não conhece nenhuma hipótese de responsabilidade do juiz em caso tal. Há, é certo, previsão de responsabilidade jurídica em alguns casos, como, por exemplo, quando o Código Civil trata dos interesses dos incapazes e do descuido com o seu patrimônio, ou quando o Código de Processo Civil prevê responsabilidade do juiz por falta de oportuna manifestação. Mas esses são isolados e de escassa ou nenhuma aplicação. Também a responsabilidade funcional é apurada diante de faltas outras que não no desempenho da liberdade de julgar. A responsabilidade política, de sua vez, está prevista apenas para os Ministros do Supremo Tribunal Federal. Por fim, a responsabilidade civil do Estado pelos atos dos juízes tem recebido sempre interpretação restritiva. Mas essas situações todas prevêem aplicação de uma sanção prevista em lei, por isso digo que são de responsabilidade jurídica.

Não havendo sanção jurídica prevista em lei, aplicável ao juiz que deixa de fazer o devido uso da cláusula geral, estamos diante de um caso de responsabilidade social. Nesta, a relação se estabelece diretamente entre o juiz e a sociedade, que se manifestará através dos diversos modos e meios de controle difuso. Para isso, é preciso em primeiro lugar garantir a publicidade e a divulgação das decisões; em segundo, contar com uma sociedade organizada, da área técnica ou não, que tenha condições de avaliar a decisão e dar a resposta. Nesse particular, exercem importante papel a imprensa, os meios eletrônicos de comunicação, os livros especializados e os periódicos, que divulgam, analisam e criticam as decisões.

$\mathrm{Na}$ verdade, a primeira e principal responsabilidade é a pessoal, pela qual o juiz é chamado a prestar contas perante sua própria consciência.

Em resumo, a cláusula geral impõe ao julgador o uso de uma técnica judicial especial de julgar, estando o seu exercício limitado pelo ordenamento constitucional o desempenho arbitrário ou omisso gera a responsabilidade social do juiz.

Quero terminar dizendo da honra que tenho em estar aqui para testemunhar a qualidade do trabalho profissional desen volvido pela Prof ${ }^{\mathrm{a}}$ JUDITH MARTINS COSTA, que enobrece esta Casa, engran dece o Curso de Mestrado e Doutorado a que pertence e que prossegue com o mesmo brilho a tradição dos Martins Costa.

A tese que escreveu para o seu doutoramento, hoje transformada em livro, teria sido motivo de especial satisfação do Prof. CLÓVIS DO COUTO E SILVA, quem tanto devemos, pelas lições e pelo exemplo de sempre procurar a excelência acadêmica, o que a Dra. JUDITH alcançou, inteligência privilegiada a serviço do Direito.

\title{
Nuevo Dinamismo o Estancamento: El futuro de las relaciones entre el Mercosur y la UE*
}

\author{
Wrich Wehnex
}

Profesor Visitante DAAD/CAPES en la Facultad de Derecho de la Universidad Federal de Rio Grande do Sul; Doctor en Derecho por la Universidad de Colonia, Alemania.

\section{Introducción}

n el transcurso del año pàsado tuvieron lugar acontecimientos importantes para las relaciones entre la Unión Europea ${ }^{1}$ y el Mercosur. Cabe destacar entre ellos el encuentro de los jefes de Estado y de Gobierno de la UE, del Mercosur y de Chile en el marco de la Cumbre de Rio en junio de 1999. También tiene importancia la entrada en vigor del Acuerdo Marco Interregional poco después de la cumbre, el 1 de junio del mismo año.

Ahora ya no son exclusivamente los juristas ibéricos que acompañan el desarollo dentro del Mercosur. En el resto de los paises europeos los autores muestran su interés en los esfuerzos de integración en el Cono Sur?2. El interes europeo se debe por un lado a los

Este trabajo no habría sido posible sin la más valiosa ayuda de la Doctora Marta Jiménez Olivar, Profesora de Derecho Internacional Público en las Universidades UFRGS y P.UC en Porto Alegre. Portanto quiero agradecerle una vez más por la colaboración productiva y agradable. El presente trabajo está basado en el artículo „EU und Mercosur: Auf dem Weg zur Freihandelszone?" del mismo autor, publicado en la revista „Recht der Internationalen Wirtschaft", № 5/2000, pág. 370 ss.

1. El término „Unión Europea (UE)" se usa aqui para hacer referencia genérica al conjunto de los 12 Estados que firmaron el Tratado de Maastricht y a los 3 Estados que se adhirieron a este Tratado en el momento de transformarse en miembros do la UE. debe tenerse en cuenta que la UE queda configurada por las tres Comunidades Europeas y otras áreas de cooperación intergubernamental ver al respecto art A pár. 3 del Trado de a Unín Europea (los articulos A a $E$ del Tratado de la Unión Europea han sido sustituidos por los artículos 1 UE a 28 UE).

2. Ver de la doctrina alemana: Renata Martins, MERCOSUR: Der Südamerikanische Gemeinsame Markt im Überblick, en: Recht der Internationalen Wirtschaft 1999, pág. 851 ss.; Jürgen Samtleben, Das internationale Prozeß- und Privatrecht des Mercosur, Rabels Zeitschrít, tomo 63 (1999), pág. 1 ss.; observando el proceso continuamente: Calixto Salomão Filho /Jürgen Samtleben, Der Südamerikanische Gemeinsame Markt - Eine rechtliche Analyse des Mercosur, en: Wertpapier Mitteilungen 1992, pág. 1345 ss., pág. 1385 ss.; Samtleben, Jürgen, Der Südamerikanische Gemeinsame Markt (Mercosur) und seine neue Verfassung, en: Wertpapier Mitteilungen 1996, pág. 1997 ss.; ibid, Das Recht des Mercosur - wichtig für Europa?, en: Europäische Zeitschritt für Wirtschaftsrecht 1998, antes de pág. 65; ibid., Las Perspectivas para un Tribunal de Mercosur y la Experiencia Europea, en:

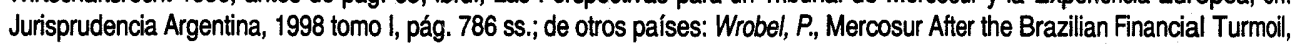
en: International Spectator 1999, Vol. 34, № 3, pág. 81 ss.; Page, S., The Relationship between the European Union and Mercosur en:International Spectator 1999, Vol. 34, № 3, pág. 91 s. Mercosur, engouement malgre les turbulences, en: Moniteur du Commerce International (MOCl) 1999, № 1377, pág. 55 ss. 
logros económicos de ese proyecto de integración latinoamericana más reciente; a pesar de las turbulencias económicas y políticas casi permanentes se reconoce el éxito económico del Mercosur en los primeros años de su existencia. Por otro lado el método de integración ha despertado el interés principalmente de los comunitaristas. Ahora bien, puede añadirse otro aspecto: Parece que las relaciones entre Europa y América Latina, practicamente paralizadas durante décadas, están experimentando una fase de revitalización ${ }^{3}$. Especialmente los representantes de la Unión Europea, del Mercosur y de Chile se pusieron de acuerdo sobre los principios básicos de su futura cooperación y expresaron su voluntad de „construir una colaboración más profunda y fructífera entre las dos comunidades" 4 .

En los paises Participantes del Mercosur se ha escrito mucho sobre los más diversos aspectos de la integración mercosureña. Pero en realidad pocos son los autores que se han ocupado detalladamente de las relaciones entre la UE y el Mercosur tomando como base los vinculos jurídicos ya existentes. Algunas valoraciones iniciales del posible acercamiento entre los dos bloques económicos fueron entusiastas. Así la celebración del Acuerdo Marco Interregional fue vista como "la aproximación más seria de Europa con América Latina desde la visita de Cristóbal

Colón"s. Sin embargo, la relación polifacética y compleja entre Mercosur y UE exije cierta cautela frente a estimaciones positivos precipitados. Ahora bien, parece imprescindible un analisis sistemático delos vínculos birregionales basado en las regulaciones actuales. Con tal motivo se debe tener en cuenta el desarollo normativo de las relaciones birregionales hasta el momento. Ya en este ámbito la polémica interna de la UE en torno a las negociaciones con el Mercosur sirve como ejemplo de una relación no siempre harmónica (II.). Un examen jurídico especialmente nítido exije la cuestión del objetivo central establecido para la cooperación entre Mercosur y UE: Por tanto nos ocupamos del proyecto destacado del Acuerdo Marco Interregional que es la creación de la llamada Asociación Interregional (III.). De gran interes no solamente es el status quo de las relaciones. Mucha importancia tienen también las perspectivas de éxito para la realización de los objetivos establecidos. Con tal motivo serán presentados algunos factores determinantes para el desarollo futuro de las relaciones birregionales (IV.). Estos factores se tomarán después para ver sus repercusiones en la relación entre el Mercosur y la UE hasta el momento (V.). Lo expuesto debe servir para sacar conclusiones para una valoración de la

observanse las repercusiones en la prensa euopea: Larraya, en: El País del 28.6. 1999, pág. 1; Hautin-Guiraut, en: Le Monde de 29.06. 1999, pág. 2; Oehrlein/Haubrich, en: Frankfurter Allgemeine Zeitung del 29.6.1999, pág. 1.

4. Reunión de Jefes de Estado y de Gobierno de Mercosur, Chile y de la Unión Europea - Comunicado Conjunto de Rio de Janeiro, del 28 de junio de 1999; EU-Doc. 9410/99 (Press 207), cifra 2.

5. Castro Escudero, Alfredo, El Mercosur, contra viento y marea, en: Comercio Exterior - Sección Latinoamericana 1994, pág. 999. cooperación actual y para indicar algunas opciones estratégicas para ambas regiones (VI.).

\section{EI desarollo de las relaciones birregionales hasta el momento}

Desde su creación en el año 1991 los Estados Partes del Mercosur no dejaron lugar a dudas que el relacionamiento con la UE sería un objetivo prioritário en su agenda económica y en su política exterior. Ya en abril del 91, poco más de un mes después de la firma del Tratado de Asunción, los ministros de asuntos exteriores del Mercosur se encontraron en Bruselas con representantes de la Comisión Europea $^{6}$. En aquel encuentro se establecieron las bases del primer paso de la cooperación interregional. Así fué firmado el Acuerdo de Cooperación Interinstitucional en el año 1992. Dos acontecimientos más marcan el empeño de la relación hasta ahora: La pieza clave de la Cooperación entre el Mercosur y la UE sigue siendo el Acuerdo Marco Interregional del año 1995. El último paso significativo lo representan los resultados del encuentro de los jefes de Estado y de Gobierno del Mercosur, de la UE y de Chile en el marco de la Cumbre de Rio.

\section{El Acuerdo de Cooperación Interinstitucional.}

El Acuerdo de Cooperación Interinstitucional representa la primera manifestación contractual en las relaciones entre la entonces CE y el Mercosur ${ }^{7}$. El acuerdo tuvo como objetivo principal transferir cierto know how en materia de integración económica de la Comunidad hacia el entonces todavía muy joven Mercosur ${ }^{8}$. Este instrumento se limitó a determina medidas de cooperación exclusivamente técnicas e administrativas entre los órganos y carecía por completo de normas materiales. Debido a su contenido limitado el acuerdo resultó rapidamente insuficiente para una aprofundización más amplia de las relaciones birregionales ${ }^{9}$. Así este instrumento de cooperación tiene actualmente un significado meramente político: La CE fué la primera a establecer oficialmente el diálogo político con Mercosur. De esa relación también surgió en forma de dicho acuerdo el primer aunque modesto - resultado concreto de los esfuerzos del Mercosur de determinar su papel en el escenario internacional.

\section{El Acuerdo Marco}

\section{Interregional de Cooperación}

El Mercosur y la UE conseguieron en diciembre del año 1995 crear una base

6. Bull. EG 4-1991, cifra 1.3.36.

7. Acuerdo del 29 de mayo de 1992, Bull. EG 5-1992, cifra 1.2.32; formalmente la relación contractual no fue establecida entre el Mercosur y la UE sino entre el Consejo del Mercado Común por parte del Mercosur y la Comisión de la Comunidad Europea. La Mercosur que carecía por ejemplo de la personalidad jurídica para firmar un acuerdo internacional.

8. Art. 1 y 2 del Acuerdo Interinstitucional de Cooperación.

9. ver la opinión de la misma Comisión Europea, Com (94) 428, S. 14 
contractual para el desarollo de sus relaciones mutuas. Se firmó el Acuerdo Marco Interregional (AMI) ${ }^{10}$. Hasta el momento este contrato es el núcleo de las relaciones birregionales.

En cuanto a su naturaleza jurídica el Acuerdo representa un buen ejemplo de la figura de los llamados acuerdos mixtos. Puede hablarse de un acuerdo mixto cuando la Comunidad Europea y sus Estados miembros firman conjuntamente el convenio ${ }^{11}$. Esto ya está explícito en la denominación del AMI que habla del „Acuerdo entre la Comunidad Europea y sus Estados Miembros, por una Parte, y el Mercado Común del Sur y sus Estados Partes, por Otra“. En el mismo sentido el AMI define las Partes del acuerdo ${ }^{12}$. El concepto del acuerdo mixto tiene la misma validad en el derecho del Mercosur. Tanto el Mercosur como sus Estados pueden actuar al mismo tiempo como signatarios de un tratado. En todo caso la razón por la cual se usa el instrumento de los acuerdos mixtos es que las materias a que se refiere el acuerdo no pueden considerarse atribuidas a la exclusiva competencia del organismo de integración ${ }^{13}$.

Por su propia estructura el acuerdo mixto presenta un inconveniente que en el caso del AMI fue objeto de una reclamación por parte del Parlamento Europeo ${ }^{14}$ : El elevado número de signatarios lleva a un igualmente elevado número de procesos de ratificación y transformación lo que causó un notable retraso para su entrada en vigor.

De manera muy apretada el AMI abarca un campo vasto de temas para la futura cooperación. El espectro temático se extiende desde la cooperación en materia de normas agroalimentarias e industriales, en materia aduanera y de la propiedad intelectual, tratando también la cooperación empresarial hasta llegar a la cooperación en los sectores de la telecomunicación y de la formación y educación ${ }^{15}$. Un espacio relativamente ámplio ocupa también la creación de un marco institucional para la cooperación birregional ${ }^{16}$

Ya solamente contrastando la diversidad del contenido con la cantidad limitada de normas se percibe facilmente que en el contenido del AMI las

10. Acuerdo Marco Interregional de Cooperación entre la Comunidad Europea y sus Estados Miembros, por una Parte, y el Mercado Común del Sur y sus Estados Partes, por Otra, del 15.12. 1995, DOCE 1996 № L 69/4; con Chile más tarde se firmó un acuerdo Común del Sur y sus Estados Part

11. Bleckmann, Der gemischte Vertrag im Europarecht, in: EuR 1976, S. 301 ff.; Carlos Francisco Molina del Pozo, Manual de Derecho de la Comunidad Europea, pág. 427.

12. Art. 32 del AMI

13. Carlos Francisco Molina del Pozo, Manual de Derecho de la Comunidad Europea, pág. 427

14. PE-Dok A4-118/96, S. 5

15. Art. 6-9, 11, 16 y 20 del AMl; el contenido del acuerdo no siempre se muestra altamente concreto - dice por ejemplo el art. 16 pár. 1: „Las Partes acuerdan establecer una cooperación común en materia de telecomunicaciones y tecnologías de la información con vista a promover su desarrollo económico y social, impulsar la sociedad de la información y, facilitar el camino hacia la modernización de la sociedad.".

16. Los art. 25-30 del AMl preven la creación del llamado Consejo de Cooperación como órgano más alto, de una llamada Comisión Mixta de Cooperación y de la llamada Subcomisión Mixta Comercial.

Revista da Faculdade de Direito da UFRGS, v. 18, 2000 estipulaciones programáticas prevalecen sobre las normas operativas y dotadas de obligaciones concretas.

Sobresaliendo de esta diversidad temática y representando el objetivo central del AMI a largo plazo se encuentra el proyecto de la creación de una Asociación Interregional entre la UE y el Mercosur ${ }^{17}$. Antes de entrar en el examen del contenido de esa figura se requiere un análisis exacto de la técnica normative al formular el objetivo final. Pues el AMI se limita a emitir una declaración de mera intención. Su objetivo no es la creación de dicha asociación, sino como reza el proprio texto „la preparación de las condiciones para la creación de una Asociación Interregional" 18 . Los contratantes no dejaron lugar a dudas que los pasos siguientes como el comienzo de negociaciones concretas - y tanto más la creación misma - requerían nuevos arreglos y también un nuevo acuerdo entre EU y Mercosur ${ }^{19}$. Con la sutileza necesária podemos decir que el objetivo del AMI no es la creación de una Asociación Interregional sino solamente la creación de un instrumento preparatorio para la preparación misma de la creación de la Asociación Interregional. En el mismo

17. la Asociación Interregional está mencionada en el preámbulo y los Art. 2 par. 1, 4, 14 par. 3, 25 par. 3, 27 par. 5 lit. dy 34 par. 2 del AMI.

18. Art. 2 pár. 1 del AMI.

19. ver tb. Art. 34 par. 2 del AMl: „Las Partes, de conformidad con sus procedimientos respectivos, y en función de los trabajos y propuestas elaboradas en el marco institucional del presente Acuerdo, determinarán la oportunidad, el momento y las condiciones para iniciar las negociaciones conducentes a la conformación de la Asociación Interregional."

20. Informe de IRELA, INF-95/5-MERC vom 14. Sept. 1995, S. 1; Núñez, Crónica de una negociación, in: Archivos del Presente Nr. 3 (1995/96), S. 124; Dromi/Molina del Pozo, Acuerdo Mercosur-Unión Europea, S. 28

21. Bull. EU 7/8-1999 cifra 1.4.149.

22. Art. 34 par. 2 del AMI. sentido se puede hablar de un acuerdo interino ${ }^{20}$ con lo cual se expresa el carácter transitório y evolutivo del AMI y del proceso de acercamiento entre Mercosur y UE.

\section{La Cumbre de Rio y la entrada en vigor del AMI}

Pocos fueron los impulsos engendrados por el AMI durante los primeros años después de su firma. Tuvieron lugar algunos encuentros de significado limitado en el marco de dos de los órganos previstos por el tratado. Sin embargo, la posibilidad de avanzar hacia la Asociación Interregional ya se veía bloqueada por el mero hecho de que el AMI todavía no estuviera en vigor. A causa de los pesados trámites de ratificación la entrada en vigor se dilató hasta el 1 de julio de $1999^{21}$.

El proceso no avanzó automaticamente con la entrada en vigor de los contratantes de determinar independientemente del AMI "la oportunidad, el momento y las condiciones para iniciar las negociaciones conducentes a la conformación de la Asociación Interregional"22. Aqui es donde encontramos la importancia de la Cumbre del Acuerdo como resultado de la decisión 
de Rio: Los representantes reunidos en Rio anunciaron el anuncio formal del inicio de las negociaciones ${ }^{23}$. Tomando como base una decisión interna de la UE sobre los detalles de las negociaciones las conversaciones sobre asuntos no arancelarios empezarían a fines del 1999 y las negociaciones arancelarias comenzarían en julio de $2001^{24}$. Poco después el Consejo de Asuntos Generales de la CE también adoptó el mandato para la Comisión Europea para iniciar las conversaciones comerciales con el Mercosur y Chile ${ }^{25}$. Así fue facilitado el primer encuentro del Consejo de Cooperación que representa el órgano más alto de las instituciones creadas por el $\mathrm{AMI}^{26}$. Esta reunión marcó el comienzo oficial para la creación de la Asociación Interregional. Los representantes acordaron la estructura, la metodología y el calendario de las negociaciones. Complementando la estructura institucional prevista por el AMI decidieron también crear nuevos órganos para llevar a cabo las negociaciones. El llamado Comité de Negociaciones Birregionales seria responsable de la conducción general y la gestión de las negociaciones. Este comité recibería el apoyo de un Subcomité de Cooperación y tendría la facultad de crear otros subgrupos.
Las próximas reuniones del Comité de Negociaciones Birregionales y de su Subcomité fueron previstas para los meses de marzo-abril 2000.

\section{Análisis jurídico del objetivo principal del AMI: La Asociación Interregional}

Frente a la decisión de Rio se impone con vehemencia la pregunta sobre el significado y el contenido de la figura de la Asociación Interregional declarada objetivo central de todos los empeños. Muchos autores consideran como núcleo del modelo de la Asociación Interregional la creación de una zona de libre comercio, es decir la eliminación de todos los aranceles y otras barreras comerciales en el comercio recíproco entre Mercosur y $\mathrm{UE}^{27}$. Esta opinión no es irrefutable, aun cuando su resultado no esté lejos de la realidad. La Asociación Interregional no contiene necesariamente una zona de libre comercio. Tal equiparación no puede fundamentarse en los conocimientos del Derecho Internacional Público o del Derecho Comunitario Europeo ni en la práctica de las asociaciones conocidas hasta el momento.

23. Reunión de Jefes de Estado y de Gobierno de Mercosur, Chile y de la Unión Europea - Comunicado Conjunto de Rio de Janeiro, del 28 de junio de 1999; EU-Doc. 9410/99 (Press 207), cifra 6.

24. Instituto de Relaciones Europeo-Latinoamericanas (IRELA), La Cumbre de Rio: ¿Hacia una asociación estratégica?, Madrid, Informe 99/8-CUM del 30.06. 1999, pág. 3.

25. Bulletin EU 9-1999 cifra 1.4.75.

26. Primer Consejo de Cooperación UE-Mercosur - Comunicado de Prensa Conjunto, 24 de noviembre de 1999, EU-Doc. 13290/99 (Press 371)

27. Matthias Herdegen, Derecho Económico Internacional, 2a Edición, Medellín 1998, § 9, cifra 1.

Revista da Faculdade de Direito da UFRGS, v. 18, 2000

1. La Asociación en el Derecho

Internacional Público

Según revela el Derecho Internacional Público ${ }^{28}$ el punto de partida para la definición del término de la asociación es la calidad de miembro de una Organización Internacional. Generalmente se entiende por asociación un vínculo a una Organización Internacional debilitado (=abgeschwächt) en comparación con el estatus de miembro de pleno derecho. La asociación otorga al asociado entre 1 y 99 por ciento de los derechos y obligaciones del miembro y tiene por objetivo facilitar la participación del asociado en los fines de la organización.

Por lo anterior se puede deducir facilmente que la UE y el Mercosur a través del proyecto de su Asociación Interregional no buscan la forma de Asociación que conoce el Derecho Internacional Público. Ahora bien, esta noción de la asociación nos proporciona solamente un elemento que es válido también para la relación que persiguen los dos bloques: $\mathrm{El}$ instrumento de la Asociación está caracterizado por la amplitud y gran variedad de su posible contenido; el término no esta susceptible de una definición general.
2. La Asociación en el Derecho Comunitario Europeo

El derecho de la Asociación conocido dentro de las Comunidades Europeas se ha alejado de la noción que de la asociación tiene el Derecho Internacional Público. El estatus de miembro como punto de contacto es abandonado. También son muy diversos los objetivos de las Asociaciones por parte de la Comunidad Europea. Las distintas formas de Asociación tienen como base jurídica el artículo 238 Tratado CE (actualmente $310 \mathrm{CE})^{29}$. A través de esa norma la Comunidad desarolló su relación frente a las antiguas colonias y otros Estados africanos, del Caribe y del Pacífico en la forma des los llamados Acuerdos de Lomé ${ }^{30}$. También la Asociación dinámica, dirigida hacia la ulterior adhesión de los asociados a la Comunidad se fundamenta en dicho articulo ${ }^{31}$. Por último la Asociación para el libre comercio y la asociación con fines de otorgar ayuda al desarollo encuentran su base jurídica en el artículo 238.

En toda la normativa comunitaria no se encuentra una determinación de las características de la figura de la Asociación. De forma genérica se habla solamente de una relación "que entrañe derechos y

28. Ignaz Seidl-Hohenveldern/GerhardLoibl, Das Recht der Internationalen Organisationen, 5. Edición 1996, §31, cifra 515; Peter Hollenweger, Die Assoziation von Staaten mit internationalen Organisationen, 1963, pág. 15.

29. Se sigue aqui al sistema uniforme de de los artículos de los Tratados en los textos del Tribunal de Justicia y del Tribunal de Primera Instancia, ver al respecto: Comunicado de Prensa del T.J., n $n^{2} 57 / 99$ del 30 de julio de 1999; para una introducción al derecho de las asociaciones en español: Carlos Francisco Molina del Pozo, Manual de Derecho de la Comunidad Europea, pág. 420 ss.

30. Una introducción proporciona: Schweitzer/Hummer, cifras 702,707

31. v. Bogdandy, en: Grabizz/Bogdandy/Nettesheim, Europäisches Außenwirtschattsrecht, 1994, pág. 18 
obligaciones recíprocos, acciones comunes y procedimientos particulares" ${ }^{32}$. La doctrina no consigue superar notablemente este nivel de ambiguedad. La asociación según un intento definitorio todavía muy eurocentrista se entiende como un vínculo permanente, general e institucionalizado, de cooperación que consagra una participación de países terceros en los objetivos comunitarios"33. También los acuerdos mismos que mencionan expresamente la creación de Asociaciones no proporcionan ninguna aclaración sistemática del contenido de esa relación.

No puede realmente sorprender que también la práctica de la Comunidad Europea en el ámbito de las Asociaciones es variada y heterogénea. En el ramo de Tratados de Asociación se encuentran meros acuerdos de cooperación con vistas a la ayuda al desarollo ${ }^{34}$, pero se halla también el establecimiento de vínculos estrechísimos para preparar el ingreso dentro de la Comunidad ${ }^{35}$. Finalmente una sóla asociación puede variar notablemente en contenido y en intensidad ${ }^{36}$.

Por lo expuesto la definición del término Asociación tiene que quedar

\section{Art. 238 Tratado CE (actualmente 310)}

33. Pescatore, Les rélations exterieures des Communautés Européennes, en: Recueil des Cours de l'Academie de Droit Internacional, 1961, vol. 11, pág. 104.; Colombo, La nature juridique de l'association a la CEE, en: L'association à la CEE, 1974, pág. 14 habla más correctamente de los „objetivos comunes“ en vez de los "objetivos comunitarios“.

34. Así aconteción en el caso de la asociación de los llamados Estados ACP, ver Gert Nicolaysen, Europarecht, Tomo 2

35. Así se puede observar en el Acuerdo que establece la asociación entre las Comunidades Europeas y la República de Polonia de 16.12.1991, DOCE 1993, № L 348, S. 1 ff.

36. Vease la asociación de la CE con Malta, establecida el 12.9. 1963, DOCE 1971 № L 61, pág. 2 que no prevía la adhesión de ese estado a la CE.

37. TJCE de 30.9.1987, Demirel, exp. $12 / 86$.

38. Ejemplo típico para esta forma de asociación son los Acuerdos de Lomé; aqui las concesiones arancelaris permanecen unilaterales, ver por ejemplo art. 130, 131 del tercero Acuerdo de Lomé del 8.12.1984, DOCE 1986 № L $86,3$. asociación prevista debe ser regida justamente por este principio de la reciprocidad. El AMI mismo habla claramente de una „liberación [...] recíproca de todo el comercio" 39

Combinando las disposiciones del AMI con las exigencias del reglamento multilateral para el comercio mundial llegamos a la conclusión que concesiones comerciales entre Mercosur y UE sólo pueden ser otorgadas si se realizan por lo menos en el marco de una zona de libre comercio.

Es bien sabido que el punto de partida del actual sistema de comercio mundial se encuentra en el General Agreement of Tade and Tariffs que fue suscrito en el año 1947 y por lo tanto se denomina GATT $47^{40}$. El principio de nación más favorecida, contenido en su articulo I del GATT 47, constituye desde entonces el núcleo del régimen del GATT. El GATT actual, conocido como GATT 94, está conformado por el original GATT 47 y las modificaciones realizadas en la Ronda de Uruguay. El resultado más visible de esa ronda de negociaciones fue la sustición del conjunto disperso de las reglas comerciales multilaterales por la Organización Mundial de Comercio $\mathrm{OMC}^{41}$. Esta es concebida como el marco institucional de las relaciones económicas

entre sus estados miembros. El acuerdo GATT 94 y los demás acuerdos cerrados al final de la Ronda de Uruguay son partes integrantes de la OMC y forman en su conjunto la normativa material del comercio internacional.

Los contratantes pretenden liberalizar su comercio y prometieron guardar la "conformidad con las reglas de la $\mathrm{OMC}^{\text {" } 42}$. De esas reglas se desprende que la única posibilidad de una liberalización comercial sin violar al mismo tiempo el principio de la nación más favorecida es justamente como mínimo la creación de una zona de libre comercio ${ }^{43}$. Otras formas de concesiones que no se extendiesen a todos los otros miembros de la OMC constituirían una infracción de la clausula de la nación más favorecida. Es porque tal principio fundamental de la $\mathrm{OMC}^{44}$ ordena que generalmente todas las ventajas comerciales tienen que ser concedidas imediatamente y sin condiciones a todos los otros miembros de la OMC. Reconociendo las ventajas de la integración las normas de la OMC permiten una excepción de la aplicación rigida del principio. Una de las condiciones inevitables para aprovechar el privilegio es que la liberalización se realice en la forma de una zona de libre comercio o de una unión aduanera.

\section{Preámbulo, inc. 9 del AMI}

40. Brand/Zamora, Basic Documents of International Law, Tomo 1, 1990, pág. 9 ss.

41. Agreement Establishing the World Trade Organization, I.L.M. 33 (1994), pág. 9 ss.; la función de marco institucional para las relaciones comerciales entre sus miembros está expresada en el Art. II inc. 1; Art. II inc. 2

42. Art. 4 y preámbulo, inc. 9 del AMI

43. Art. XXIV (5) a (10) del GATT 94

44. La clausula de la nación más favorecida se encuentra en el art. 1 del GATT 94 
A pesar del hecho que una liberalización comercial se deba realizar en forma de una zona de libre comercio o aún de unión aduanera Mercosur y UE optaron por el término de la Asociación Interregional. Así evitaron minuciosamente el uso de una forma de cooperación que implicase ya per definitionem concesiones comerciales. No antes de la Cumbre de Rio empezaron a abandonar esa práctica cautelosa. Ahí los representantes de los dos bloques acordaron explicitamente „entablar negociaciones entre Mercosur, Chile y la Unión Europea para la liberalización bilateral, progresiva y recíproca del comercio sin excluir ningún sector “45. En cuanto a las relaciones birregionales en las negociaciones Chile debe ser tratado como si fuese miembro del Mercosur. En la forma de un single undertaking los resultados de las negociaciones entre los tres constituirán un conjunto único y indivisible ${ }^{46}$.

\section{Factores determinantes para el desarollo futuro de las relaciones birregionales}

Después del análisis técnico de la configuración para el desarollo de las rela-

45. Reunión de Jefes de Estado y de Gobierno de Mercosur, Chile y de la Unión Europea - Comunicado Conjunto de Rio de Janeiro, del 28 de junio de 1999; EU-Doc. 9410/99 (Press 207), cifra 6 .

46. Reunión de Jefes de Estado y de Gobierno de Mercosur, Chile y de la Unión Europea - Comunicado Conjunto de Rio de Janeiro, del 28 de junio de 1999; EU-Doc. 9410/99 (Press 207), cifra 6.

47. María Angeles Benítez, Relaciones entre la Unión Europea y el Mercosur, in: Miguel Angel Ciuro Caldani, Del Mercosur, Buenos Aires 1996, S. 53; Roberto Dromi/Carlos Molina del Pozo, Acuerdo Mercosur-Unión Europea, Buenos Aires 1996, S. 20; Comisión Europea comparte esta posición, COM (94) 428, pág. 7

48. Así lo estipula la Comisión Europea, Doc. Com (97) 2000, pág. 38

49. Instituto de Relaciones Europeo-Latinoamericanas (IRELA), Las Relaciones entre Europa y América Latina: Hacia una agenda birregional para el siglo XXI, Informe Especial „Cumbre de Rio, Madrid, pág. 4; el préambulo del AMI comienza con las palabras: "Considerando los profundos lazos historicos, culturales, políticos y económicos que les unen e inspirados en los valores comunes a sus pueblos". Con texto identico el Comunicado Conjunto de Rio de Janeiro, del 28 de junio de 1999. EU-Doc. 0410/99 (Press 207), cifra 1.

Revista da Faculdade de Direito da UFRGS, v. 18, 2000 regiones todavía se ha prestado poca atención al aspecto de los ordenamientos jurídicos. El empresario europeo reencuentra en los estados del Mercosur y en Chile vários elementos de la tradición jurídica europea bien $\operatorname{conocidos}^{50}$. Las codificaciones civiles fueron - con variaciones - influenciadas por el Code Civil francés y recurren con frecuencia al derecho italiano, alemán y suizo ${ }^{51}$. También las cuatro legislaciones societárias dentro del Mercosur responden a sistemas jurídicos homogéneos, de raíz romano germánica ${ }^{52}$. Los estados del Mercosur no sólo avanzan en la supresión de barreras comerciales sino también cabe resaltar ciertos éxitos en el campo de la harmonización dè sus legislaciones nacionales ${ }^{53}$. Por lo tanto va creciendo la posibilidad para los inversores y empresarios europeos de aprovechar un espácio económico común y un espácio jurídico común. De todos modos en Europa las cuatro libertades que proporcionaría la construcción de un mercado común son bien conocidas y gozan de un alto reconocimiento ${ }^{54}$.

Los lazos históricos y culturales entre los pueblos del Mercosur y de la UE tanto como los valores comunes no pueden ser negadas. Sin embargo se debe dudar la viabilidad de ésta visión de los vínculos. Será en primer lugar la realidad económica que decidirá sobre el éxito o el fracaso de las relaciones entre el Mercosur y la UE.

Desde el punto de vista económico el Mercosur se muestra definitivamente atractivo y ya ha obtenido éxitos significativos $^{55}$. El conjunto de los estados puede ser considerado una de las nuevas zonas emergentes y representa un mercado potencial de aproximadamente 200 millones de consumidores. Los estados del Mercosur son responsables por un $50 \%$ del BIP latinoamericano. La UE es el primer socio comercial del Mercosur. Si a principios

50. Esto se limita desde luego a los ordenmientos juridicos del continente europeo

51. Konrad Zweigert/Hein Kötz, Einführung in die Rechtsvergleichung, pág. $112 \mathrm{ss}$

52. Miguel Carlos Araya, El derecho de sociedades en el Mercosur, in: Luis Antonio Velasco San Pedro (Hrsg.), Dos Modelos de Integración Económica, Valladolid 1998, S. 151; Guiomar T. Estrella Faria, As sociedades comerciais e a formaçāo dos blocos econômicos de naçōes, en: Maristela Basso (Hrsg.), Mercosur - Seus efeitos jurídicos, económicos e políticos nos Estados-

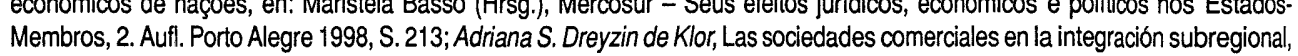
Membros, 2. Aufl. Porto Alegre 1998, S. 213; Adriana S. Dreyzin de Klor, Las sociedades comerciales
en: Revista de Derecho Privado y Comunitario - Concurso y Quiebra I, NN 10, 1996, pág. $487 \mathrm{~s}$.

53. Ver los esfuerzos del Mercosur en el área del DIPr que abarcan tanto el sector de los aspectos sustanciales y el que se refiere a las cuestiones de carácter procesal, ocupandose tanto de la parte general como de la parte especial del DIPr, Fernández Arroyo, La nueva configuración del Derecho Internacional Privado del Mercosur, en: Revista de Derecho del Mercosur, № 4 (1999), páa 38 ss.; Samtleben, Das internationale Prozeß- und Privatrecht des Mercosur, Rabels Zeitschritt, tomo 63 (1999), pág. 1 ss.

54. Estas son la libre circulación de bienes, de personas, servicios y capitales, ver en el Derecho Comunitario: art. 9-36 Tratado CE (actualmente art. 23-31 CE) 48-58 Tratado CE (actualmente art. 39-48 CE) 48-58 Tratado CE (actualmente art. 39-48 CE)

67-73 Tratado CE (actualmente art. 56-60 CE); objetivos similares se encuentran en el art. 1 pár. 2 del Tratado de Asunción.

55. para las relaciones comerciales entre la UE y América Latina y el Mercosur: Eurostat, European Union/Latin America - Caribbean Summit, Key figures - Relations between the European Union and the Latin American - Caribbean countries, Special Memo del 3.5. 1999; IRELA Las Relaciones entre Europa y América Latina: Hacia una agenda birregional para el siglo XXI, Informe Especial "Cumbre de Rio, Madrid, pág. 12; Cifra l.1. de la "Rio Declaration of the des Mercosur European Union Business Forum“ del 23.2. 1999, puede ser consultada a través de: http://mww.eurosur.org/eurosur 

libre Comercio de las Américas (ALCA). estados americanos persigue el de la década el Mercosur absorbía alrededor de un tercio de las ventas totales de la UE a América Latina, esta proporción ahora supera el $50 \%$. Este hecho se torna más significativo todavía teniendo en cuenta que el crecimiento de las exportaciones de la UE a Latinoamerica es uno de los más altos en total ${ }^{56}$. Ya hoy dia un $40 \%$ de las IED realizadas en el Mercosur se radican en los paises de la UE. Especialmente en los primeros años de su existencia el volumen del comercio dentro del Mercosur disparó ${ }^{57}$.

El Mercosur puede vivir otro aumento de importancia para Europa por los esfuerzos para la creación de la Área de A initiativa de los E.E.U.U. un total de 34 establecimiento de una zona de libre comercio de Alaska hacia Tierra de fuego ${ }^{58}$. En su última reunión los ministros de económia de los estados participantes reafirmaron la voluntad de concluir el proyecto a más tardar en el año 2005 y decidieron también lanzar discusiones sobre la estructura general de un acuerdo del ALCA $^{59}$. El éxito de los esfuerzos convertiría el Mercosur para la UE en una

de las regiónes clave para el acceso y la participación en esta zona de libre comercio.

\section{La estructura del comercio birregional - Problemas de la PAC}

Mirando sólo la situación descrita anteriormente las circunstancias parecen favorables a un desarollo positivo de las relaciones comerciales entre UE y Mercosur. Además la UE se confiesa oficialmente partidaria de la liberlización comercial y de una postura liberal. Así figura como objetivo de la Política Comercial Común de la CE contribuir „al desarollo armonioso del comercio mundial, a la supresión progresiva de las restricciones a los intercambios internacionales y a la reducción de las barreras arancelarias ${ }^{\text {"60 }}$. Pero cabe destacar que la participación en el comercio mundial no es para nada un proyecto altruista. En el marco de la Política Comercial Común los Estados Miembros por un lado intentan también realizar uno de sus intereses primordiales que es es fortalecimiento de la posición europea en la lucha por la competencia en el mercado mundial. Por el otro lado a la UE no le parece incompatible con sus principios venerables

56. En el período del 1988 hasta el 1998 sólo las exportaciones de la UE hacia los países de Europa Central y del Este crecieron a una tasa más alta, ver: Eurostat, Millennium Round - The European Union Figures for the Seattle Conference, Memo 9/99 del

57. Peña, El cuadro institucional de las relaciones entre la Europa Comunitaria y el Mercosur, en: Aintegração aberta - Um projecto da União Europeia e do Mercosul, Lissabon 1995, pág. 222; Iñigo Febrel Melgarejo, La integración económica de Mercosur,en: Boletín Económico de ICE, № 2516, 1996, pág. 25.

58. La decisión de crear el ALCA fue tomada en la cumbre de 34 jefes de estado y de gobierno americanos en diciembre del 1994 en Miami, International Legal Materials (I.L.M.) 1995, pág. 808 ss.; en la cumbre de avril 1998 se decidió de seguir el proyecto manteniendo los plazos previstos, I.L.M. 1998, pág. 947 ss.

59. Véase la declaración de los 34 ministros de economia en su reunión en Toronto del 4.11. 1999, consigase a través de http:// www.ftaa-alca.org.

60. Art. 110 par. 1 Tratado CE (actualmente $131 \mathrm{CE}$ ) 18.11.1999, pág. 6. de guardar sus intereses justamente a través de la protección de ciertos sectores. Destaca como ejemplo de esta práctica la Política Agricola Común (PAC) de la CE ${ }^{61}$. La PAC conoce como instrumento más comunitario y más significativo el establecimiento de las Organizaciones comunes de mercado. Generalmente una Organización común de mercado se basa en un mecanismo de intervención para garantizar vários niveles de precios fijados. También forma parte un sistema de protección exterior que se basa en la compensación de precios. Intentando igualar el nivel de precios comunitarios con los del mercado mundial los productos provenientes de terceros estados pueden ser gravadas para situar su precio por encima del precio comunitario. A menudo este objetivo se alcanza también a través de las llamadas restituciones a la exportación que consisten en pagar al agricultor una ayuda que le permite exportar sus productos más caros al precio del mercado mundial.

En vista del masivo interés de la UE en su producción agroalimentaria es la estructura de las relaciones comerciales con el Mercosur que difiçulta la liberalización comercial: Por la estructura actual de los intercambios comerciales para la UE la liberalización del comercio prometería altas ventajas para el sector de bienes de consumo, capital y servicios. Es en particular el Brasil que considera que no están suficientemente preparados para una apertura de los mercados. La liberalización afectaría tanto la producción brasileña como las exportaciones a los otros paises del Mercosur. Por el otro lado para los socios del Mercosur sería altamente ventajosa una apertura del mercado europeo para los productos agrícolas. Estos ya representan juntos con los productos pesqueros más de la mitad de sus ventas totales-a la UE y cuyo potencial de expansión de exportaciones es significativo $^{62}$.

Ahora bien, los intentos de liberalizar el comercio birregional entran automaticamente en conflicto con las medidas de la UE en el marco de la PAC. El proyecto de apertura recíproca de los mercados enfrenta así un obstaculo agrícolas de la CE se pone de manifesto sabiendo que alrededor del 70 por 100 del presupuesto comunitario se dedica a financiar esta política comunitaria ${ }^{63}$.

\section{Las reglas de la OMC}

La ansiedad de la UE frente a la apertura de sus mercados se vé forzada por las reglamentaciones alcanzadas ahora a algunos de los sectores industriales todavia inmenso. La magnitud de las actividades

61. Los fundamentos de la PAC se encuentran en los art. 38-47 Tratado CE (actualmente art. 32-38 CE); para una introducción ver: Joane Scott, Tragic Triumph: Agricultural Trade, the Common Agricultural Policy and the Uruguay Round, en: Nicholas Emilioul David O'Keefe, The European Union and World Trade Law, New York u.a. 1996, pág. 165 ss.; Molina del Pozo, Manual de Derecho de la Comunidad Europea, pág. $733 \mathrm{ss}$; Eberhard Grabitz/Armin von Bogdandy/Martin Nettesheim, Europäisches Außenwirtschaftsrecht - Der Zugang zum Binnenmarkt: Primärrecht, Handelsschutz und Außenaspekte der Außenwirtschaftsrecht - Der Zugang zum
Binnenmarktharmonisierung, 1994, pág. $147 \mathrm{ss}$.

62. estructura y intereses según: Instituto de Relaciones Europeo-Latinoamericanas (IRELA), La Cumbre de Rio: ¿ Hacia una asociación estratégica?, Madrid, Informe 99/8-CUM del 30.06. 1999, pág. 14.

63. Molina del Pozo, Manual de Derecho de la Comunidad Europea, pág. 733 
nivel multilateral. Para la UE una liberalización comercial especialmente en el sector agrícola ya se tornó inevitable en el marco de la OMC. Por primera vez en el sistema multilateral la Ronda de Uruguay trajo obligaciones significativa de eliminar barreras comeciales en el sector agroalimentario para la UE ${ }^{64}$. También en el marco de la llamada Ronda del Milenio de la OMC la UE no podrá evitar concesiones comerciales en el campo agrícola.

En la construcción de las relacione birregionales cobra importancia otro elemento del sistema multilateral. Ya vimos que una de las condiciones bajo los cuales los estados y grupos de estados pueden gozar de excepciones del principio de la nación más favorecida es la adopción de una zona de libre comercio. Pero el GATT exige otro requisito para obtener este privilegio: $\mathrm{La}$ eliminación de las barreras arancelarias y no tarifarias tiene que ser ampliada a la totalidad del comercio (substantially all the trade) en su espacio económico ${ }^{65}$. Las normas de la $\mathrm{OMC}$ autorizan sólo a excluir temporalmente de los acuerdos de libre comercio un 10 por 100 del comercio total ${ }^{66}$. Por un lado resulta positivo que el Mercosur y la UE no puedan excluir ningún sector de un futuro programa de liberalización

comercial. Pero el sistema de la OMC abre como única alternativa alternativa a la liberalización de substantially all the trade la posibilidad de abstenerse por completo de un tratamiento preferencial.

\section{Repercusión de los factores determinantes en las relaciones birregionales}

Los factores determinantes analizados en el párrafo anterior se han plasmado claramente en el desarollo de las relaciones birregionales hasta el momento. Sobre todo la UE ha mostrado su cautela en el momento de contraer obgligaciones concretas. Esta postura se pone de manifesto en el esfuerzo de evitar escrupulosamente el uso del término de la zona de libre comercio en los documentos oficiales. En la propuesta de la Comisión de las Comunidades Europeas para el mandato de negociación del AMI se habló todavia de una zona de libre comercio. Pero fue el Consejo de ministros de la UE que excluyó este término del mandato finalmente aprobado ${ }^{67}$. Por lo tanto el modelo de una Asociación Interregional entre el Mercosur y la UE no expresa una originalidad especial al definir el objetivo central de las relaciones. El proyecto tampoco refleja el deseo de los contratantes

64. La CE está obligada a bajar el nivel de sus compensaciones de precios en un 20 por 100, las restituciones a la exportación en un 36 por 100, el volumen total de las exportaciones subvencionadas debe ser reducido por 21 por 100, según: Scott Joane Scoth Tragic Triumph: Agricultural Trade, the Common Agricultural Policy and the Uruguay Round, en: Nicholas Emiliou/David O'Keefe, The European Union and World Trade Law, New York u.a. 1996, pág. 165 ss.

65. Art. XXIV (8) (a) y (b) del GATT 94

66. IRELA, Las Relaciones entre Europa y América Latina: Hacia una agenda birregional para el siglo XXI, Informe Especial „Cumbre de Rio, Madrid, pág. 15.

67. Mercedes Olmedo Gavilán/Antonio Gómez-Crespo López, Relaciones UE-Mercosur, en: Boletín Económico de ICE № 2489 (1996), pág. 54. de establecer relaciones económicas especialmente estrechas. El análisis del significado de la asociación en el Derecho Internacional Público y en el Derecho mostró sobretodo que las partes eligieron este modelo por su contenido ámplio y poco concreto. La decisión de crear una Asociación Interregional es en primer lugar una medida para no contraer en un momento temprano obligaciones jurídicamente vinculantes.

En cuanto al sector agrícola en Europa no faltan avisos de las amenazas para los intereses europeos que pueden resultar de la liberalización del comercio con el Mercosur ${ }^{68}$. Por eso también 'los documentos de cooperación contienen el aviso que una liberalización del comercio birregional sólo se debe realizar „teniendo en cuenta la sensibilidad de ciertos productos"69. Fueron además estos productos sensibles del sector agrario que casi condujeron al fracaso de la Cumbre de Rio: La decisión del Consejo de asuntos generales de comenzar negociaciones comerciales con Mercosur había sido paralizada principalmente a iniciativa francesa, alegando las amenazas para la agricultura europea. Un compromiso fue

alcanzado prácticamente en el último momento. A esas querellas se debe también que la UE pretende excluir de las negociaciones los aspectos arancelarios hasta julio de $2001^{70}$. En este compromiso la cautela aplicada por la UE se manifiesta desde una doble vertiente. No sólo se dilató el comienzo de las negociaciones arancelarias. También se evitó fijar plazos concretos para el desarollo y la finalización de las negociaciones. Esta práctica ya fué aplicada en el caso del AMI mismo y tanto el Comunicado Conjunto de Rio como los resultados del primer encuentro en el seno del Consejo de Cooperación no preveen nada concreto al respecto.

En las relaciones entre los dos bloques repercuten también las obligaciones creadas en el marco de la OMC y la recién comenzada Ronda del Milenio. El comunicado de Rio subraya tres vezes el significado de la OMC para la creación de la Asociación Interregional entre el Mercosur y la UE ${ }^{71}$. Dentro de la UE prevalece la opinión que el desarollo de las relaciones con el Mercosur debe ser vinculado estrechamente con los resultados de la Ronda del Milenio. La conclusión de las negociaciones en el marco de la OMC representa una condición para un acuerdo

68. DOCE 1995 № C 151, pág. 31 cifra 7; ver también el rechazo de un posible acuerdo de libre comercio entre UE y Mercosur por organizaciones agrícola españolas, en: El País del 5.7.1999, 8; con intenciones similares la interpelación parlamentaria al Consejo de la UE, DOCE 1999 № C. 135, pág. 202

69. así el preámbulo del AMl; Comunicado Conjunto de Rio de Janeiro, del 28 de junio de 1999; EU-Doc. 94 10/99 (Press 207), cifra 4; repetido en: Primer Consejo de Cooperación UE-Mercosur - Comunicado de Prensa Conjunto, 24 de noviembre de 1999, EUDoc. 13290/99 (Press 371).

70. Instituto de Relaciones Europeo-Latinoamericanas (IRELA), La Cumbre de Rio: ¿Hacia una asociación estratégica?, Madrid, Informe 99/8-CUM del 30.06. 1999, pág. 3; Abbelard, en: Le Monde del 29.6.1999, Suplemento „Focus“, pág. V|; Larea, en: E Intorme 99/8-CUM del 30.06. 1999, pág. 3; Abbelard, en: Le Monde del 29.6.1999, Suplemento
País del 29.6.99, pág. 1; Fickinger, en: Frankfurter Allgemeine Zeitung del 30.6.99, pág. 15.

71. Comunicado Conjunto de Rio de Janeiro, del 28 de junio de 1999; EU-Doc. 9410/99 (Press 207), cirras 4, 6, 8.

Revista da Faculdade de Direito da UFRGS, v. 18, 2000 
de libre comercio con el Mercosur ${ }^{72}$. Frente a esa decisión ya se percebe cierto disentimiento. Últimamente la UE repitió su postura de limitar las negociaciones primero a los aspectos generales en el comercio mutuo. Por el contrario el Mercosur entiende que esa cuestión debe ser discutida en el seno del Comité de Negociaciones Birregional ${ }^{73}$.

\section{Conclusiones: Valorización y perspectivas de las relaciones birregionales}

Lo que parece justo decir de algunos aspectos de la integración mercosureña caracteriza también las relaciones entre el Mercosur y la UE: El futuro desarollo está marcado por la incertidumbre. El Mercosur mismo en cambio ya dispone de diversos éxitos económicos y políticos. Aunque sus estados rechazan la implementación de elementos supranacionales y la pérdida de soberanía, el modelo de integración ha avanzado. El sistema integracionista flexible muchas vezes se muestra adecuado para paises que están sujetos a oscilaciones macroeconómicas hoy por dia desconocidas en el ámbito de la UE. En general es menester aplicar mucha cautela en el momento de valorizar el proceso del Mercosur usando el modelo europeo como punto de referencia. Tanto la integración dentro del Mercosur como la evaluación de

la integración mercosureña por parte de los europeos necesitan un enfoque suficientemente independiente para ser éxitosas.

El conjunto de los logros y atractivos económicos aboga por un desarollo pósitivo de las relaciones birregionales. En este contexto le corresponde al Mercosur presentar la mayor estabilidad posible. Su atractivo depende notablemente de su desarollo económico y todavía más de sus progresos en el camino hacia un auténtico mercado común. Resulta óbvio que un mercado estable y consolidado representa una parte negociadora mucho más atraente que un conjunto de estados oscilando sin rumbo.

El acercamiento entre la UE y el Mercosur que se manifestó entre otras en la Cumbre de Rio es un primer éxito en las relaciones birregionales. Sin embargo los logros concretos para el comercio entre los bloques son todavía extramemente pequeños. Posibles ventajas para la economía europea pueden deribar de momento principalmente de la dínamica del Mercosur mismo. La facilitación del comercio birregional a través de las relaciones institucionales entre el Mercosur y la UE sólo pueden ser esperadas a largo plazo. Sobre todo el proteccionismo agrícola europeo frena un desarollo más positivo. Una última indicación del obstáculo que

72. Las conclusiones de los ministros de asuntos exteriores con la ocasión de la Cumbre de Rio son citadas en: Le Monde del 29.06 . 1999: nles] négociatins seront conduites et clôturées en tenant dûment compte des résultats du cycle (du millénaire) de l'Organisation mondiale du commerce (OMC)[..."; ; los ministros de asuntos exteriores de la UE hasta declararon que las negociaciones con el Mercosur y Chile „doivent être conclus après les négociations du cycle de l'OMC".

73. Primer Consejo de Cooperación UE-Mercosur - Comunicado de Prensa Conjunto, 24 de noviembre de 1999, EU-Doc. 13290/99 (Press 371). representa el sector agricola y del significado de la estructura comercial son las negociaciones de la UE para un acuerdo de libre comercio con México. Estas a diferencia del proceso lento que viven el Mercosur y la UE se llevan a cabo rápidamente. Los progresos se deben en gran parte al hecho que el sector agrícola represente sólo cerca del 7 por 100 de las exportaciones mexicanas a la UE ${ }^{74}$.

Aún cuando no se comparte la clasificación de la PAC como ,anacrónica y absurda"75 se puede decir que los problemas surgiendo de la misma son „más reservas de tipo psicológico que de contenido real" ${ }^{\text {"76 }}$ y por lo tanto deberían ser superables. La observación de la falta de un fondo real de los excesos de la PAC se encuentra reforzada por el hecho que el conjuntro de los paises de la UE desde hace mucho tiempo se aleje de ser una región basada en la agricultura ${ }^{77}$.

Otro problema está vinculado con la PAC de la Comunidad Europea. Se trata del establecimiento de un nexus estrecho entre concesiones comerciales para el Mercosur y los resultados de la recien comenzada Ronda del Milenio. Parece altamente probable que el mantenimiento de una vinculación estricta equivaldría al fracaso de las negociaciones entre Mercosur y UE. Esto lo indica tanto el comienzo conflictivo de la Ronda de Seattle como las experiencias de la última Ronda de negociaciones. La Ronda de Uruguay que había comenzado en el año 1986 debería primero terminar en el año 1990, después en el 1992 y su finalización no se produjo hasta el año $1994^{78}$. Suponiendo una evolución parecida en el caso de la Ronda del Milenio es de esperar una reorientación del Mercosur que le alejaría de la UE partiendo rumbo al ALCA y los EUA; los empresarios europeos estarían al mismo tiempo amenazados de perder su standing todavia positivo en la región principalmente a los EUA. Tomando el conjuto de América Latina las exportaciones estadounidenses ya triplican las europeas ${ }^{79}$.

Sin embargo son justamente esos últimos factores que pueden ser usados como instrumentos para un desarollo más positivo de la cooperación birregional. El desarollo de las relaciones depende también de la habilidad de los estados del Mercosur a la hora de aprovechar su posición y su peso como conjunto. La realización de una zona de libre comercio transamericana junto con la posibilidad de un acercamiento a los E.E.U.U. son opciones estratégicas del Mercosur para presionar a la UE. Alusiones por parte del Mercosur de dar una nueva

74. En cuanto al desarollo de las negociaciones con Méilco: IRELA, Las Relaciones entre Europa y América Latina: Hacia una agenda birregional para el siglo XXI, Informe Especial "Cumbre de Rio, Madrid, pág. 13.

75. Así lo expresó el antiguo presidente argentino Carlos Menem, citado según: Relea/Larraya, El País del 30.6. 1999, pág. 2

76. Comentario del Comisario Manuel Marín a las problemas con la posición francesa, según: El País del 22.6. 1999, pág. 2.

77. La participación de los productos agroalimentarios en el comercio total de la UE va constantemente diminuyendo, ver: „Millennium Round" - The European Union Figures for the Seattle Conference, Eurostat, Memo 9/99, pág. 3

78. Thomas Oppermann, Die Europäische Gemeinschaft und Union in der Welthandelsorganisation (WTO), en: Zentrum tür Europäisches Wirtschaftsrecht (Editora), Vorträge und Berichte, № 54/1999, pág. 37.

79. El País del 30.6. 1999, pág. 2. 
prioridad a su relacionamiento con los del Mercosur no se encuentren entre los E.E.U.U. en comparación con la socios comerciales más importantes de la cooperación transatlantica se presentan $U^{80}$ los europeos no se pueden permitir la como una posibilidad notable de forzar su falta de estar economciamente ausentes en posición negociadora. Aunque los estados el Mercosur.

\section{RESENHA}

80. „Millennium Round“ - The European Union Figures for the Seattle Conference, Eurostat, Memo 9/99, pág. 6 